\title{
Institutionen als Ingardensche intentionale Gegenstände
}

\author{
Daniel von Wachter
}

Leitseite des Autors: http://von-wachter.de

Epost: ersetze "ABC” in daniel@ABC.de durch “von-wachter"

Adresse: Dr. Dr. Daniel von Wachter, Institut für Philosophie, Universität

München, PF 42, D-80539 München.

Dies ist ein Vorabdruck von:

Wachter, Daniel von. 2005. „Institutionen als Ingardensche intentionale Gegenstände. "In Institutionen und ihre Ontologie, Hg. G. Schönrich. Heusenstamm: Ontos Verlag, 61-72.

Dieses Dokument ist erhältlich auf http://epub.ub.uni-muenchen.de. (Format A5; Empfehlung: 2 Seiten pro Blatt drucken)

Den Bienenzuchtverein München e.V., der das Ziel hat, die Bienenhaltung im Raum München zu fördern, gäbe es wahrscheinlich nicht, wenn es keine Bienen gäbe. Ganz sicher ist das nicht, denn es könnte ja sein, daß es zwar keine Bienen, aber die Idee von Bienen gibt und sich mit ihr ein Verein zur Erzeugung von Bienen bildet. Ganz sicher ist aber, daß es den Verein nicht gäbe, wenn es keine Menschen gäbe. Nicht nur deshalb, weil der Verein dann keine Mitglieder hätte, sondern auch deshalb, weil nur mit Geist begabte Wesen Vereine bilden können. Moleküle können Kristalle bilden, Bäume können Blüten hervorbringen, aber Dinge, die keinen Geist haben, können keine Vereine bilden. 
Der polnische Philosoph Roman Ingarden (1893-1970) hat uns in seinem vierbändigen Riesenwerk zur Ontologie Der Streit um die Existenz der Welt einen reichen ontologischen Steinbruch hinterlassen, in dem wir auch Gegenstände finden, die nicht existierten, gäbe es keine mit Geist begabten Wesen. Institutionen sind ein Beispiel solcher Gegenstände, die Ingarden „intentionale Gegenstände“ nennt. In diesem Aufsatz werde ich von Ingardens Ansatz ausgehend verschiedene Arten von Institutionen unterscheiden und insbesondere untersuchen, von was für geistigen Vorgängen diese Institutionen genau abhängen.

\section{Arten der Seinsabhängigkeit}

Im ersten Band des Streit, dem er den Titel „Existentialontologie“ gibt, unterscheidet Ingarden verschiedene „Seinsweisen“, z.B. Realsein und Idealsein. Ingarden meint also, daß es verschiedene Arten von Sein gibt und daß „,ist“ daher vieldeutig ist. (Ingarden 1964, 66) Ein idealer Gegenstand, das ist ein unzeitlicher Gegenstand, z.B. eine Zahl, eine Idee oder ein Universale (von Ingarden „ideale Qualität“ genannt). Daß ein Gegenstand ein idealer ist, liegt daran, daß er auf eine bestimmte Weise existiert. Ein realer Gegenstand hingegen, das ist ein zeitlicher Gegenstand wie z.B. ein Stein. Wenn ein Gegenstand ein realer ist, dann ist auch alles an ihm und sind auch alle seine Teile real. Zum Beispiel sind gemäß Ingarden auch die Eigenschaften eines Steines (die Ingarden als individuell und als Exemplifizierungen von Universalien auffaßt) real.

An einer Seinsweise, meint Ingarden, seien verschiedene Aspekte zu unterscheiden, sog. „existentiale Momente“. Dazu gehören insbesondere verschiedene Arten der Seinsabhängigkeit. Daß A von B seinsabhängig ist, heißt grob gesagt, daß A nicht ohne B existieren kann. Die Art der Seinsabhängigkeit, die für uns hier von Bedeutung ist, nennt Ingarden „Seinsheteronomie“. Doch zuerst seien die drei anderen Arten der Seinsabhängigkeit genannt, die Ingarden im Band I des Streit erörtert. 
Erstens unterscheidet Ingarden seinsselbständige von seinsunselbständigen Entitäten. (Ingarden 1964, \$14) (Statt Entität im Sinne von etwas Seiendes sagt Ingarden auch „Gegenständlichkeit“.) Eine seinsunselbständige Entität ist eine, die nur zusammen mit bestimmten anderen Entitäten, in der Einheit eines Ganzen existieren kann. Gemeint ist damit die Weise, wie z.B. eine Eigenschaft eines Dinges nie alleine, sondern immer nur als Eigenschaft eines Dinges mit bestimmten anderen Eigenschaften existieren kann. Unter der „Einheit eines Ganzen“ versteht Ingarden also die Einheit wie sie bei den Eigenschaften eines konkreten Dinges besteht. Jener Apfel dort hat eine Masse von $100 \mathrm{~g}$. Diese Eigenschaft kann nicht alleine, als frei schwebende Masse existieren. Sie kann nur zusammen mit einer Dichte, einer Temperatur etc., an einem Ding existieren.

Zweitens unterscheidet Ingarden seinsursprüngliche von seinsabgeleiteten Gegenständlichkeiten. Eine seinsursprüngliche Gegenständlichkeit ist eine, die „ihrem Wesen nach durch keine andere Gegenständlichkeit geschaffen werden kann“ (Ingarden 1964, 87). Sie kann auch durch nichts vernichtet werden und existiert immerwährend. Gott z.B., wenn es ihn gibt, ist eine seinsursprüngliche Entität.

Drittens unterscheidet Ingarden seinsunabhängige von seinsabhängigen Entitäten. Eine seinsabhängige Entität ist eine seinsselbständige Entität, die zu ihrem Fortbestehen „die Existenz einer anderen seinsselbständigen Gegenständlichkeit wesensmäßig erfordert“ (Ingarden 1964, 121). Als Beispiel einer seinsabhängigen Entität nennt Ingarden einen menschlichen Organismus, der seinsabhängig ist von der Existenz einer Wärmequelle, welche die Temperatur herstellt, die er zum Leben benötigt. Ein naheliegenderes Beispiel wäre ein von Gott geschaffener Gegenstand. Wenn es einen Gott gibt, dann ist jedes materielle Ding, z.B. ein Stein, seinsabhängig, weil es nur fortbestehen kann, wenn Gott es erhält.

Für unsere Untersuchung der Institutionen von Bedeutung ist die vierte Unterscheidung, die zwischen seinsautonomen und seinsheteronomen Entitäten. Eine seinsautonome Entität ist eine, die ihr „Seinsfundament außerhalb ihrer selbst hat" (Ingarden 1964, 79). Der Spezialfall, der In- 
garden und uns hier am meisten interessiert, ist der des intentionalen Gegenstandes $^{1}$, d.h. eines Gegenstandes, der von Bewußtseinsvorgängen abhängt. Eine intentionale Gegenständlichkeit ist eine, „die ihr Sein und ihr gesamtes Beschaffensein aus dem Vollzug eines auf eine bestimmte einheitliche Weise beinhalteten intentionalen Bewußtseinserlebnisses schöpft und ohne diesen Vollzug überhaupt nicht existieren würde“. Ingarden hält Institutionen für intentionale Gegenstände (Ingarden 1964, 83f), widmet ihnen aber weit weniger Aufmerksamkeit als einem anderen Beispiel intentionaler Gegenstände, nämlich den fiktiven, erdichteten Gegenständen, wie z.B. die Romanfigur Antonie Buddenbrook einer ist. (Ingarden 1965, Kap. 9; 1931) Bevor ich mich einer genaueren Untersuchung der Institutionen zuwende, möchte ich daher kurz Ingardens Ontologie der erdichteten Gegenstände vorstellen.

\section{Erdichtete Gegenstände}

Sehen wir uns die drei Hauptmerkmale erdichteter Gegenstände an: 1. ihre Bestimmungen sind nicht immanent, 2. sie haben „Unbestimmtheitsstellen“, 3. sie weisen eine „Doppelseitigkeit“ auf.

Antonie Buddenbrook (auch "Tony“ genannt) ist ein seinsheteronomer, genauer gesagt ein intentionaler Gegenstand. Das heißt nach Ingarden, daß er sein Seinfundament außerhalb seiner selbst hat. Erläuternd sagt er: „Eine Gegenständlichkeit hat ihr Seinsfundament in sich selbst, wenn sie in sich selbst etwas immanent Bestimmtes ist." Damit meint er folgendes.

\footnotetext{
1 Ingarden verwendet also „intentionaler Gegenstand“ anders als Husserl, der darunter einen Gegenstand versteht, auf den ein Bewußtseinsvorgang, ein intentionaler Akt gerichtet ist. Wenn Müller den Eifelturm sieht, ist nach Husserl der Eifelturm der intentionale Gegenstand von Müllers Wahrnehmungserlebnis. Der Eifelturm ist aber kein intentionaler Gegenstand in Ingardens Sinn, denn er hat sein Seinsfundament in sich selbst. Was Ingarden einen intentionalen Gegenstand nennt, ist in etwa das, was Husserl einen „bloß intentionalen Gegenstand“ nennt, z.B. den Gott Jupiter, den Müller sich vorstellt. Vgl. Husserls V. Logische Untersuchung und (Wachter 2000, 71-74).
} 
Antonie Buddenbrook hat graublaue Augen. Das ist wahr. Aber irgendwie unterscheidet sich ihr Graublaue-Augen-Haben von meinem Graublaue-Augen-Haben. Daß ich graublaue Augen habe, ist wahr, weil es da Augen aus Fleisch und Blut gibt, die Licht bestimmter Wellenlänge reflektieren. Daß der Satz „Antonie Buddenbrook hat graublaue Augen“ wahr ist, liegt an nichts dergleichen. Es gibt nirgends Augen aus Fleisch und Blut, von denen der Satz handelt und die Licht reflektieren. Der Satz ist nur deshalb wahr, weil Thomas Mann in seinem Roman „Die Buddenbrooks" geschrieben hat, daß Antonie graublaue Augen hat. In diesem Sinne sagt Ingarden, daß dieser Gegenstand sein Seinsfundament nicht in sich selbst, sondern in bestimmten geistigen Vorgängen Thomas Manns hat. Auch spricht er von der „Nichtimmanenz der Bestimmtheiten“ (Ingarden 1964, 82) eines solchen Gegenstandes: das Graublausein der Augen Antonies ist eine Bestimmtheit des Gegenstandes, aber es ist dem Gegenstand nicht immanent, d.h. es hängt ab von etwas, das nicht ein Teil des Gegenstandes ist.

Verschiedene Arten intentionaler, seinsheteronomer Gegenstände kann man unterscheiden nach der Art von Geistesereignissen, von denen sie abhängen. Was ist das also für eine Art von Geistesereignissen, von denen erdichtete Gegenstände abhängen? Ingarden spricht davon, daß einem erdichteten Gegenstand seine Bestimmungen „bloß zugewiesen“, „vermeint“ seien (Ingarden 1964, 84). Der betreffende geistige Akt des Autors ist nicht ein Glauben, es ist ein Akt anderer Art als Thomas Manns Glauben (Überzeugtsein, Meinen), daß seine Frau blaue Augen habe. Die Bestimmung hängt auch nicht von dem Glauben anderer $a b$, daß Antonie Buddenbrook graublaue Augen habe. Die geistigen Akte, von denen erdichtete Gegenstände abhängen, sind von einer eigenen Art, wir können sie Akte des Erdichtens nennen. Der Autor greift unter den geistig konstruierbaren Szenarien eines heraus und macht es zu dem im Kunstwerk bestehenden Szenario. Dies geschieht Schritt für Schritt. Zu einer Zeit war Antonie Buddenbrook schon geschaffen, aber es stand noch nichts über ihre Augenfarbe fest. Vielleicht gab es schon das typische Zittern ihrer Oberlippe, als der Autor dann zum ersten Mal von ihren graublauen Au- 
gen schrieb. So ist eine Bestimmung der Antonie Buddenbrook nach der anderen entstanden. Der Akt ist ein Ausdenken und ein dichterisches Festlegen, z.B. durch Niederschreiben im Manuskript.

Auch nach Abschluß des betreffenden Kunstwerkes bleibt für viele mögliche Bestimmungen unbestimmt, ob sie auf den Gegenstand zutreffen. Ob Tony einen Leberfleck am linken Schulterblatt hat, ist unbestimmt, denn es steht nichts darüber im Buch. Ob Thomas Mann hingegen einen Leberfleck am linken Schulterblatt hatte, ist eine zu entdeckende Tatsache. Das liegt daran, daß Thomas Mann ein seinsautonomer Gegenstand ist, während Tony ein erdichteter Gegenstand ist. Ingarden nennt Bestimmungen, von denen für einen bestimmten seinsheteronomen Gegenstand auf diese Weise nicht feststeht, ob sie auf ihn zutreffen, „Unbestimmtheitsstellen“.

Das dritte Hauptmerkmal erdichteter Gegenstände ist nach Ingarden die „Doppelseitigkeit“ ihres Aufbaus (Ingarden 1965, 211-218). Auf der einen Seite hat ein erdichteter Gegenstand einen „Gehalt“; dazu gehören alle ihm dichterisch zugeschriebenen Eigenschaften, wie z.B. das Graublausein von Tonys Augen. Auf der anderen Seite hat ein erdichteter Gegenstand eine „intentionale Struktur“; dazu gehört, daß der Gegenstand einen Gehalt hat, daß er ein intentionaler Gegenstand ist oder daß er z.B. von Thomas Mann erschaffen ist.

Nach Ingarden zeichnen sich erdichtete Gegenstände also dadurch aus, daß ihre Eigenschaften nur dichterisch zugeschrieben sind, daß sie Unbestimmtheitsstellen haben und daß sie doppelseitig sind. Das halte ich für eine richtige Analyse erdichteter Gegenstände. Ingarden scheint jedoch anzunehmen, daß alle intentionalen Gegenstände, also auch Institutionen so sind; und das halte ich nicht für ganz zutreffend. Daher werde ich nun andere Arten von intentionalen Gegenständen und insbesondere von Institutionen davon unterscheiden. Besonderes Augenmerk möchte ich dabei darauf legen, in welcher Art von geistigen Vorgängen das Seinsfundament jeweils liegt. Welches also sind die geistigen Vorgänge, auf denen Vereine, Ehen, Stiftungen, die bayerische Flagge, etc. gründen? Hat John 
Searle recht, wenn er über Institutionen sagt, sie existierten nur, weil wir glauben, sie existierten? (Searle 1995, 1)

\section{Nicht-darstellende Geistesgebilde}

Von den erdichteten Gegenständen sind zunächst Gebilde wie Beethovens Neunte Symphonie oder das Völkerrecht zu unterscheiden. Dies sind nicht-darstellende Geistesgebilde, will sagen es sind nicht Gegenstände, denen Eigenschaften dichtend zugeschrieben werden, die sonst von seinsautonomen Gegenständen getragen werden. Die Prädikate, mit denen diese Gegenstände beschrieben werden, haben keine Anwendung im Bereich der seinsautonomen Gegenstände. Das Prädikat „Allegro maestoso“ zum Beispiel wird nur im Bereich der musikalischen Werke angewendet. Das Material, aus dem nichtdarstellende Geistesgebilde bestehen, entstammt nicht dem Bereich der seinsautonomen Gegenstände.

Der fundierende geistige Vorgang der nichtdarstellenden Geistesgebilde ist dem der erdichteten Gebilde ähnlich, unterscheidet sich aber darin, daß er nicht Prädikate aus dem Bereich der seinsautonomen Gegenstände verwendet, sondern eigene. Wir können diese geistigen Vorgänge nichtdarstellendes geistiges Schaffen nennen.

Gesetze, die auch Ingarden für intentionale Gegenstände hält (Ingarden 1964, 83), sind allesamt nicht-darstellende Geistesgebilde. Dabei ist es gleichgültig, ob die Gesetze gut oder verwerflich sind und ob sie Pflichten hervorbringen. Daß ein Gesetz verwerflich ist, heißt, daß es Handlungen vorschreibt, deren Unterlassung Pflicht ist. Ein Beispiel wäre ein Gesetz, welches allen vorschreibt, ihre behinderten Kinder zu töten. Manche Gesetze schreiben Handlungen vor, die durchzuführen ohnehin Pflicht wäre, z.B. seine Kinder nicht zu töten. Andere Gesetze schreiben Handlungen vor, die nicht Pflicht wären, gäbe es das Gesetz nicht. Unter bestimmten Umständen - wenn der Gesetzgeber entsprechend qualifiziert ist und das Recht hat, das betreffende vorzuschreiben - kann ein Gesetzgeber eine Pflicht hervorbringen; ähnlich wie auch Eltern ihren Kindern manches zur Pflicht machen können, wenn es nicht zu viel verlangt ist etc. So 
kann zum Beispiel ein Gesetz es zur Pflicht machen, an einem Zebrastreifen anzuhalten, wenn dort ein Passant die Straße überqueren möchte. Gäbe es das Gesetz nicht, bestünde keine solche Pflicht. In jedem Falle sind Gesetze nicht-darstellende Geistesgebilde. Menschen denken sie sich aus und erlassen sie.

Das, was Ingarden als „Doppelseitigkeit“ bezeichnet, gibt es bei den nichtdarstellenden Geistesgebilden auch; man kann nämlich einerseits zwischen dem Gehalt des Gebildes, also z.B. den Notentext bzw. die damit definierte Musik, und andererseits Eigenschaften des Gebildes, wie daß es im Jahre 1803 entstanden ist oder daß es von Franz Schubert oder vom Verkehrsminister Dollinger geschaffen wurde.

Unbestimmtheitsstellen, wie bei den erdichteten Gegenständen, gibt es bei den nicht-darstellenden Geistesgebilden nicht, zumindest nicht in der gleichen Weise. Der Grund dafür ist, daß es bei nicht-darstellenden Geistesgebilden keinen dargestellten Gegenstand gibt, bei dem es feststünde, ob das betreffende Prädikat zutrifft. Bei einem Menschen ist es etwas zu Entdeckendes, ob er einen Leberfleck auf dem linken Schulterblatt hat. Die Romanfigur Tony stellt einen Menschen dar, daher entsteht die Frage, ob sie so einen Leberfleck hat. Bei nicht-darstellenden Geistesgebilden gibt es diese Situation nicht, aber manchmal eine ähnliche. Dann nämlich, wenn dem Gegenstand eine Bestimmung zukommen könnte, es aber nicht festgelegt ist, ob sie ihm zukommt. Ein Gesetz zum Beispiel, das Schülern öffentlicher Schulen verbietet, ein Kopftuch zu tragen, könnte so sein, daß es unbestimmt ist, ob es nur Muslims oder ob es allen verbietet, ein Kopftuch zu tragen. ${ }^{2}$ (Mehr zu Gesetzen und Pflichten unten.) Unbestimmtheitsstellen im engeren Sinne gibt es nur bei erdichteten Gegenständen. Im weiteren, gerade beschriebenen Sinn kann es Unbestimmtheiten aber bei allen Arten intentionaler Gegenstände geben.

Die Grenzen des Begriffes einer Institution sind vage, aber weder erdichtete Gegenstände noch nicht-darstellende Gegenstände bezeichnen wir normalerweise als Institutionen. Die intentionalen Gegenstände der im

\footnotetext{
$2 \mathrm{Zu}$ Unbestimmtheitsphänomenen bei musikalischen Werken siehe (Ingarden 1986).
} 
folgenden unterschiedenen vier Arten lassen sich jedoch alle unter den Begriff einer Institution fassen. Diese Arten sind: Symbolinstitutionen, Gruppeninstitutionen, moralische Institutionen und Spiele. Ich schließe nicht aus, daß manche Gegenstände zu mehreren dieser Arten gehören. Das ist dann der Fall, wenn ein Gegenstand durch geistige Vorgänge der betreffenden verschiedenen Arten fundiert ist.

\section{Symbolinstitutionen}

Auch Geld und Flaggen sind intentionale Gegenstände. Wir können sie Symbolinstitutionen oder institutionelle Artefakte nennen. Weitere Beispiele sind: die bayerische Flagge, die Ortsschilder von Dresden, die Torlinien eines Fußballfeldes oder das Fischsymbol der ersten Christen in den Katakomben. Institutionelle Artefakte sind Gegenstände, gewöhnlich materielle Gegenstände, die eine Bedeutung oder eine Funktion haben, die sie nicht hätten, wenn es bestimmte geistige Vorgänge nicht gäbe. Die Vorgänge nämlich, die Ingarden als das Seinsfundament diese Gegenstände bezeichnen würde. John Searle nimmt auf diese geistigen Vorgänge m.E. richtig Bezug, indem er sie ausdrückt durch Sätze der Form „X gilt als Y unter Umständen C“ (Searle 1995, 28). Menschen haben also die Fähigkeit, materielle Gegenstände mit Funktionen $\mathrm{zu}$ belegen. Diese Funktion ist dann so lange in Kraft wie die Umstände entsprechend sind und wie genug und die richtigen Leute die Funktion und die Bedeutung anerkennen. Wir können diese geistigen Vorgänge „Funktionszuweisungen"nennen.

Anders als die meisten erdichteten Gegenstände und nicht-darstellenden Geistesgebilde haben viele institutionelle Artefakte Exemplare. Eine finnische 1-Euro-Münze zum Beispiel gibt es nicht nur einmal, sondern es gibt viele davon, und es können bei Bedarf noch mehr Exemplare hergestellt werden.

Eine Doppelseitigkeit kann man auch bei institutionellen Artefakten feststellen. Einerseits gibt es da einen Gehalt; z.B. den Wert der Münze von 1 Euro oder die Zusage, daß man für den Geldschein auf Anfrage bei der 
Zentralbank soundsoviel Gold bekäme. Andererseits sind da die natürlichen Eigenschaften des Dinges, z.B. das Gewicht der Münze.

\section{Gruppeninstitutionen}

Was wir gewöhnlich als „Institutionen“ bezeichnen, ist eine weitere Art intentionaler Gegenstände: Universitäten, Regierungen, Bandenführer, Stiftungen, Vereine, das Bundesfinanzministerium, oder Initiationsriten. Wir können hier von „Gruppeninstitutionen“ sprechen. Von was für geistigen Vorgängen sind diese Sachen abhängig? Es ist eine eigene Art geistiger Vorgänge, die verschieden ist von den geistigen Vorgängen, die institutionellen Artefakten zugrundeliegen. Damit eine Stiftung beispielsweise existieren kann, müssen in einer Gesellschaft genug und die richtigen Menschen die Idee einer Stiftung kennen und bestimmte Mechanismen für das Errichten kennen und anerkennen. Zumindest müssen sie eine Stelle anerkennen, die für die Errichtung oder Genehmigung von Stiftungen zuständig ist. Diese Stelle kann ein Mensch oder wiederum eine Gruppeninstitution sein. Sowohl Anerkennung als auch Macht kann eine Stelle in die Lage versetzen, Stiftungen wirksam zu genehmigen. Ist sie weithin anerkannt als berechtigt zur Genehmigung von Stiftungen, ist das hinreichend für die Möglichkeit, Stiftungen zu genehmigen. Wenn sie sehr mächtig ist und die Möglichkeit hat, Menschen Geld wegzunehmen, kann sie Stiftungen wirksam anerkennen unabhängig von der Anerkennung durch viele weitere Menschen.

Den fundierenden geistigen Vorgang von Gruppeninstitutionen nenne ich institutionelles Anerkennen. Searle weist auch hier die entscheidende Rolle Akten der Form „X gilt als $\mathrm{Y}$ in C“ zu. Ich halte dagegen, daß nur institutionelle Artefakten durch geistige Vorgänge dieser Art begründet werden und daß der fundierende geistige Vorgang von Gruppeninstitutionen anders ist.

Bei Gruppeninstitutionen gibt es Exemplifizierungen. Zum einen kann derselbe Typ von Gruppeninstitution mehrfach exemplifiziert sein. Zum Beispiel kann es beliebig viele Exemplare vom Typ selbständige Stiftung 
geben. Zum anderen kann es innerhalb einer Gruppeninstitution Funktionen oder Ämter geben, die von Menschen eingenommen werden und die $\mathrm{zu}$ verschiedenen Zeiten von verschiedenen Menschen eingenommen werden. Die Menschen, die eine Funktion in einer Gruppeninstitution einnehmen, nenne ich „Funktionäre“.

Gruppeninstitutionen sind nicht doppelseitig, sondern dreiseitig. Bei einer bestimmten Stiftung zum Beispiel ist da zum ersten die Form der Institution, z.B. die Form „selbständige Stiftung“. Zum zweiten ist da der Gehalt der Institution; z.B. daß der Vorstand aus drei Funktionären besteht, einem Vorstand, einem Sekretär und einem Kämmerer, und daß das Ziel der Stiftung die Förderung der Metaphysik in der deutschen Philosophie ist. Zum dritten sind da Tatsachen wie die, daß Max Meier Vorstand der Stiftung im Jahre 1997 war und daß die Stiftung den Druck eines Buches über Universalien und Verursachung mit 3000 Euro gefördert hat. Wir können diese dritte Seite die „Exemplifizierungstatsachen“ nennen.

\section{Moralische Institutionen}

Institutionen wie Bruderschaften (wo man sich ewige Loyalität schwört) oder Ehe werden manchmal in die gleiche Kategorie wie Vereine gesteckt, also zu den Gruppensinstitutionen gezählt. Mit ihnen hat es jedoch etwas Besonderes auf sich. Sie bestehen nämlich durch Rechte und Pflichten, die durch geistige Vorgänge hervorgebracht sind. Ich nehme zwei Arten geistiger Vorgänge an, die unter Umständen Rechte und Pflichten hervorbringen, nämlich Versprechen und Gebote.

Die Institutionen, die ich „moralische Institutionen“ nenne, existieren, weil bestimmte Rechte und Pflichten existieren, die wegen bestimmter geistiger Vorgänge bestehen und ohne diese Vorgänge nicht bestünden. Eine Ehe besteht wesentlich deshalb, weil bestimmte Rechte und Pflichten bestehen. Beide haben die Pflicht, in guten wie in schlechten Zeiten bis zum Tod zueinander zu halten, füreinander und für die Familie zu sorgen, keine sexuellen Kontakte mit anderen Menschen zu haben, etc. Diese 
Rechte und Pflichten bestehen, weil die beiden Partner entsprechende Versprechen abgegeben haben. Der geistige Vorgang des Versprechenabgebens $^{3}$ ist deshalb ein Seinsfundament der Ehe.

Ähnlich bringen auch manche Gebote Rechte und Pflichten hervor, nämlich dann, wenn der Gebietende entsprechend qualifiziert ist. Zum Beispiel können Eltern durch Gebot für ihre Kinder die Pflicht hervorbringen, nach dem Essen das Geschirr abzuspülen. Solche Pflichten könnten ebenfalls Institutionen hervorbringen. Beispiele sind allerdings schwer $\mathrm{zu}$ finden.

Moralische Institutionen sind wie erdichtete Gegenstände zweiseitig. Sie haben einerseits einen Gehalt und andererseits Eigenschaften wie daß das Versprechen am 12. Mai gegeben wurde.

\section{Spiele}

Von den genannten intentionalen Gegenständen können wir schließlich Spiele unterscheiden. Spiele sind den Gruppeninstitutionen verwandt, indem auch sie auf einer Art Anerkennen gründen. Sie scheinen mir jedoch eine andere Art von Anerkennen zu benötigen. Sie benötigen Menschen, die die Spielregeln nicht nur kennen und anerkennen, sondern die auch mitspielen. Ein Verein braucht Menschen, die den Verein und seine Regeln anerkennen. Ein Spiel hingegen braucht Mitspieler. Was die anderen machen, ist egal. Wenn der Torwart in einem Fußballspiel vom Feld geht, um noch Milch zu kaufen, bevor die Geschäfte zumachen, erkennt er zwar vielleicht die Regeln an, aber er ist ein Spielverderber.

Bei Spielen liegt ähnlich wie bei Gruppeninstitutionen eine Dreiseitigkeit vor. Es gibt einen Gehalt, das sind die Spielregeln. Dann gibt es Eigenschaften eines Spiels wie etwa, wann es erfunden wurde. Und schließlich gibt es Exemplifizierungstatsachen, wie daß Max Müller der Torwart ist.

\footnotetext{
3 Eine tiefe Untersuchung des Vorganges des Versprechenabgebens findet sich in (Reinach 1913, 147-158).
} 


\section{Schluß}

Institutionen sind intentionale Gegenstände, d.h. ihre Existenz hängt von geistigen Vorgängen ab. In Ingardens Worten: die geistigen Vorgänge sind das Seinsfundament dieser Gegenständlichkeiten. Verschiedene Arten von Institutionen unterscheiden sich durch die Art der geistigen Vorgänge, von denen sie abhängen. Für die Existenz einer Institution ist es nicht hinreichend, daß es Menschen mit der Überzeugung gibt, daß die Institution existiere. Symbolinstitutionen sind von Funktionszuweisungen abhängig, Gruppeninstitutionen von institutionellem Anerkennen, moralische Institutionen von Versprechensakten und Spiele vom Mitspielen.

\section{Literatur}

Ingarden, Roman. 1931. Das literarische Kunstwerk. 4. Aufl. Tübingen: Niemeyer, 1972.

-_- 1964. Der Streit um die Existenz der Welt I: Existentialontologie. Tübingen: Niemeyer.

_-_. 1965. Der Streit um die Existenz der Welt II/1: Formalontologie. Tübingen: Niemeyer.

-_- 1986. The Work of Music and the Problem of its Identity. London: Macmillan.

Reinach, Adolf. 1913. Die apriorischen Grundlagen des bürgerlichen Rechts. In Sämtliche Werke, Hg. K. Schuhmann und B. Smith. München: Philosophia Verlag, 1989, 141-278.

Searle, John R. 1995. The Construction of Social Reality. London: Penguin Books. Wachter, Daniel von. 2000. Dinge und Eigenschaften: Versuch zur Ontologie. Dettelbach: Verlag J.H. Röll. 